\title{
Le statut du latin écrit de l'Antiquité en étymologie héréditaire française et romane
}

\author{
Maggiore, Marco \\ ATILF (CNRS \& Université de Lorraine) \\ giacomogramer@gmail.com \\ Buchi, Éva \\ ATILF (CNRS \& Université de Lorraine) \\ eva.buchi@atilf.fr
}

\section{Introduction}

Les dictionnaires étymologiques ont coutume de décrire l'origine des unités lexicales à travers une multitude de marqueurs catégoriels, voire de classifications discursives, sans se soucier de leur hiérarchisation conceptuelle ${ }^{1}$. Cela vaut même pour ce que l'on peut considérer comme le meilleur dictionnaire étymologique en un volume du français (Bloch \& Wartburg 1968 $)$, où l'on relève par exemple :

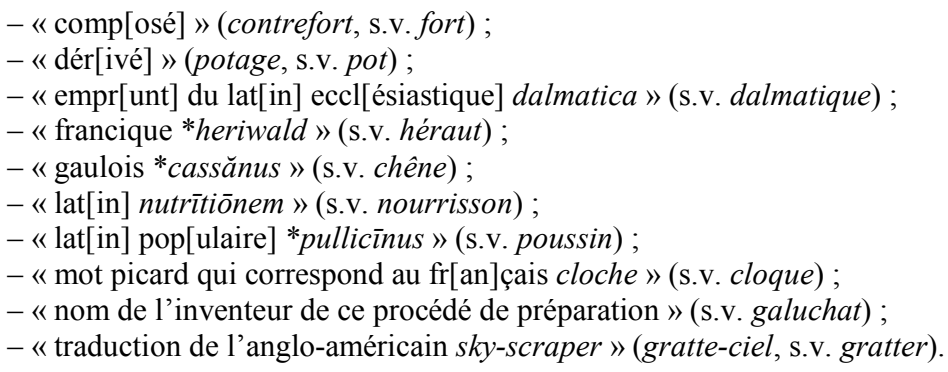

La modélisation étymologique du lexique d'une langue naturelle recommande toutefois de ramener cette multitude de cas particuliers à trois grandes classes, dont chacune exige une méthodologie propre : le lexique héréditaire, les emprunts (dont les calques forment une sous-classe) et les créations internes ( $c f$. Buchi à paraître : 4). Parmi les exemples ci-dessus, chêne, héraut, nourrisson et poussin relèvent ainsi du lexique héréditaire, cloque, dalmatique et gratte-ciel, des emprunts et contrefort, galuchat et potage, des créations internes (en l'occurrence, françaises).

Comme le suggère le syntagme étymologie héréditaire (par ellipse d'étymologie du lexique héréditaire) de son titre, cet article porte sur la première de ces trois classes étymologiques : le lexique héréditaire. Le propre de cette classe, dans le domaine roman (et donc français), c'est qu'elle forme, depuis le lancement du Dictionnaire Étymologique Roman (DÉRom) en 2008, l'objet d'un débat paradigmatique âprement disputé. En effet, tandis que les protagonistes du DÉRom proposent de conduire désormais l'étymologie héréditaire romane, à l'instar de l'étymologie héréditaire des autres familles linguistiques du monde ( $c f$. par exemple Fox 1995), au sein du paradigme de la reconstruction comparative (Buchi 2010b; 2012 ; 2013a ; Buchi, Chauveau, Gouvert \& Greub 2010 ; Buchi, González Martín, Mertens \& Schlienger à paraître ; Buchi \& Schweickard 2009; 2011a ; 2011b; Chambon à paraître), de grandes figures de la romanistique (Kramer 2011 ; Möhren 2012 ; Vàrvaro 2011a ; 2011b) critiquent vivement cette orientation théorique et militent pour un retour au paradigme classique, fondé sur les données du latin écrit. Afin de donner un rapide aperçu des différences qui caractérisent les résultats de ces deux approches méthodologiques qui coexistent désormais en étymologie romane, nous mettons ci-dessous côte à côte les étymons proposés pour quelques lexèmes héréditaires du français d'une part par le Romanisches Etymologisches Wörterbuch $\left(\mathrm{REW}_{3}\right)$ de Meyer-Lübke, qui représentera l'approche classique, d'autre part par le DÉRom : 


\begin{tabular}{|c|c|c|}
\hline Lexème français & Étymon du $\mathrm{REW}_{3}$ & Étymon du DÉRom \\
\hline clamer & $\begin{array}{l}\text { clāmāre [v.intr./tr.] 'appeler ; } \\
\text { nommer' }\end{array}$ & $\begin{array}{l}\text { */'klam-a-/ v.intr./tr./pron. } \\
\text { 'crier ; nommer ; s'appeler' }\end{array}$ \\
\hline$d i x$ & dëcěm [num. card.] 'dix' & */'deke/ num. card. 'dix' \\
\hline écouter & *ascŭltāre [v.tr.] 'écouter' & $\begin{array}{l}\text { */es'kult-a-/ v.tr. 'écouter ; } \\
\text { suivre' }\end{array}$ \\
\hline faim & fames/*famine [s.f.] 'faim' & $\begin{array}{l}\text { */'фamen/ s.n. 'faim ; famine ; } \\
\text { désir' }\end{array}$ \\
\hline février & februarius [s.m.] 'février' & */фe'Brari-u/ s.m. 'février' \\
\hline herbe & hĕrba [s.f.] 'herbe" & */'zrb-a/ */'cr $\beta$-a/ s.f. 'herbe' \\
\hline
\end{tabular}

Tableau 1 : comparaison des étymons de l'étymologie classique et de l'étymologie reconstructive

Ces quelques exemples auront suffi pour montrer que les deux méthodes n'aboutissent pas aux mêmes résultats, que cela concerne les propriétés phonologiques des étymons (dix, écouter, faim, février, herbe), leurs propriétés sémantiques (clamer, écouter, faim) ou leurs propriétés morphosyntaxiques (clamer, faim). La discussion paradigmatique actuelle ne représente donc pas une guerre de chapelles stérile, mais comporte de réels enjeux scientifiques. En même temps, les étymons mis en avant par les défenseurs de l'une et de l'autre méthode présentent quand même de grandes similitudes. Cela a amené récemment l'une d'entre nous (Buchi 2013c: 4) à utiliser la métaphore de la déclinaison magnétique pour décrire la différence entre les deux approches - tels le nord magnétique et le nord géographique, les résultats de recherche des deux méthodes sont assez proches, mais clairement distincts -, et à appeler déclinaison étymologique l'écart entre les résultats de recherche de la méthode traditionnelle et ceux de la reconstruction comparative.

Les tenants de la reconstruction comparative ont amplement communiqué sur les raisons qui les amènent à écarter le témoignage du latin écrit pour l'établissement des étymons du lexique héréditaire roman (notamment Benarroch 2013; Buchi 2010b ; 2012; 2013a; 2013c; Buchi \& Schweickard 2013; Chambon 2007 ; 2010 ; à paraître). On se contentera ici de citer le texte fondateur de Jean-Pierre Chambon (Chambon $2010: 64)$ :

\section{[...] les mots du latin écrit de l'Antiquité ne sauraient être placés à l'origine des mots héréditaires du français ou des autres langues (gallo)romanes (pas plus que le latin des textes ne peut être tenu pour l'ancêtre de ces langues elles-mêmes). Le seul moyen de faire venir à l'existence l'étymon (oral) d'un mot héréditaire est de le reconstruire sur la base de la comparaison entre formes orales affines, c'est-à-dire dont on a préalablement montré qu'elles étaient reliées par un ensemble de correspondances phoniques régulières.}

Néanmoins, il ne peut pas être question de jeter le bébé avec l'eau du bain : les témoignages massifs du latin écrit dont dispose le romaniste représentent une richesse extraordinaire, et le placent dans une situation extrêmement favorable, voire unique. Appliquée à la matière romane, la reconstruction comparative n'amène donc nullement à évacuer les données du latin écrit ; le tout est de les utiliser à bon escient, c'est-à-dire de les faire intervenir à l'étape appropriée du processus de recherche, au demeurant assez complexe. Dès lors, on peut se demander quel est le statut du latin écrit de l'Antiquité en étymologie héréditaire romane (et, par voie de conséquence, française). C'est à cette question que notre réflexion tente d'apporter une réponse.

Selon les dires de Steven Dworkin, «practitioners of Romance etymology are blessed (or cursed ?) by having at their disposal the rich documentation afforded by written Latin » (Dworkin à paraitre : 7). 
Faisant nôtre cette double assertion, nous articulerons notre réflexion en deux volets : nous montrerons d'abord (ci-dessous 2) en quoi les données du latin écrit de l'Antiquité peuvent constituer une malédiction pour l'étymologie héréditaire française et romane, puis (ci-dessous 3) en quoi elles peuvent aussi constituer une bénédiction. Dans les deux parties, l'analyse d'exemples concrets, tirés de la pratique du DÉRom, permettra d'illustrer notre propos.

\section{Le latin écrit comme malédiction}

L'étymologie du verbe quérir illustrera la malédiction que le latin écrit de l'Antiquité peut constituer - et a constitué de façon structurelle au sein du paradigme traditionnel - pour l'étymologie héréditaire.

L'approche traditionnelle considère en général fr. quérir v.tr. 's'efforcer de trouver, chercher' (dp. 1327, Klein 1961 : 154) comme une création interne du français, issue par changement de flexion verbale du français/oïlique (wallon, picard, normand, poitevin, bourbonnais, champenois, lorrain, franc-comtois) querre 'id.' (dp. fin $11^{\mathrm{e}}$ s., Gdf) :

- «seit dem Beginn des 15. J[ahr]h[undert]s belegte Neubildung für a[lt]fr[an]z[ösisch] querre » (Gamillscheg $1928^{1}-1969^{2}$ )

- « réfection, relevée au XV ${ }^{\mathrm{e}} \mathrm{s}$ [iècle], de l'a[ncien] fr[ançais] querre » (Bloch \& Wartburg $1968^{5}$ [Bloch \& Wartburg $1932^{1}: \ll[\ldots]$, relevée vers $\left.\left.1327,[\ldots] »\right]\right)$;

- « anc[ien] fr[ançais] querre [...], par changement de conjugaison » (Dauzat, Dubois \& Mitterand 2007 [déjà même analyse dans Dauzat $\left.1938^{1}\right]$ );

- « im gallorom[anischen] hat das verbum seit dem 13. j[a]h[rhundert] allmählich seine konjugation gewandelt » (von Wartburg 1945 in FEW 2, 1410a, QUAERĔRE);

- « issu, par changement de conjug[aison], de l'a[ncien] fr[ançais] querre 'chercher' » (TLF 1990);

- « réfection par changement de conjugaison (fin XII ${ }^{\mathrm{e}} \mathrm{s}\left[\right.$ iècle]) du verbe querre » (Robert historique $1992^{1}-1998^{2}$ );

- «réfection de l'a[ncien] fr[ançais] querre » (Baumgartner \& Ménard 1996).

Seule Picoche $\left(1971^{1}\right.$-2002) semble à première vue échapper à cette règle, mais en dernière analyse, sa formule "quérir [...] (querre jusqu'au $\mathrm{XV}^{\mathrm{e}} \mathrm{s}$ [iècle]) : quaerěre » renvoie à la même analyse sousjacente : quérir serait issu - selon des modalités à préciser, par exemple par analogie avec la classe flexionnelle en -ir-de querre.

En réalité, l'analyse des étymologistes n'est pas originale : elle remonte au moins - sous la forme d'une variante - à la Grammaire des langues romanes de Meyer-Lübke, où on lit que quérir a été « substitué à l'ancienne forme querre sous l'influence de férir» (Meyer-Lübke 1895: 2 : § 121), et la Phonétique française des Bourciez affirme : "quérir n'apparait qu'au XIV siècle, et doit être de création tardive pour afr. querre (= quaerěre) » (Bourciez \& Bourciez 1967 : 81).

Si l'on se positionne du point de vue du seul français, cette analyse peut convaincre : un changement de flexion du type -re vers le type -ir est tout à fait envisageable pour l'ancien français, et les datations respectives (querre: fin $11^{\mathrm{e}}$ s. ; quérir: 1327) s'accommodent parfaitement du sens de l'évolution postulée. Ce qui pourrait toutefois étonner, dans cette hypothèse, ce sont les parallèles de quérir dans d'autres parlers de la Galloromania ( $c f$. la formule de von Wartburg): une lecture même rapide de l'article QUAERERE du FEW fait apparaître que non seulement les dialectes oïliques, mais aussi le francoprovençal et l'occitan, partagent le type ‘quérir ` avec le français (FEW 2, 1408ab). L'hypothèse traditionnelle sous-entendrait-elle que ces parlers ont tous emprunté le lexème au français ? Le FEW ne répond pas à cette question, voire ne la pose pas.

Or, traiter une étymologie héréditaire au niveau du seul français, ou même au niveau de la Galloromania, représente une erreur méthodologique grave : c'est la Romania qui constitue le cadre adéquat (Buchi 2010a : 46-48). Dès lors, il convient d'établir la liste des cognats de fr. quérir, qui sont au nombre de huit (cf. Maggiore 2012/2013 in DÉRom s.v. */'kuer-e-/) :

- italien septentrional cherire $\left(1^{\text {ère }}\right.$ moitié $13^{\mathrm{e}}$ siècle-1274);

- frioulan cirî (dp. ca 1416);

- ladin chirì (dp. 1763); 


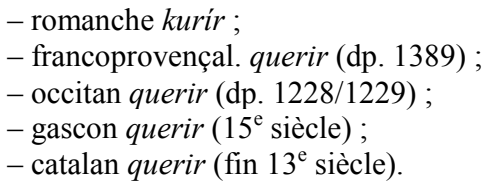

Ce type morphologique montre une distribution aréale compacte et centrale ( $c f$. Maggiore à paraître) :

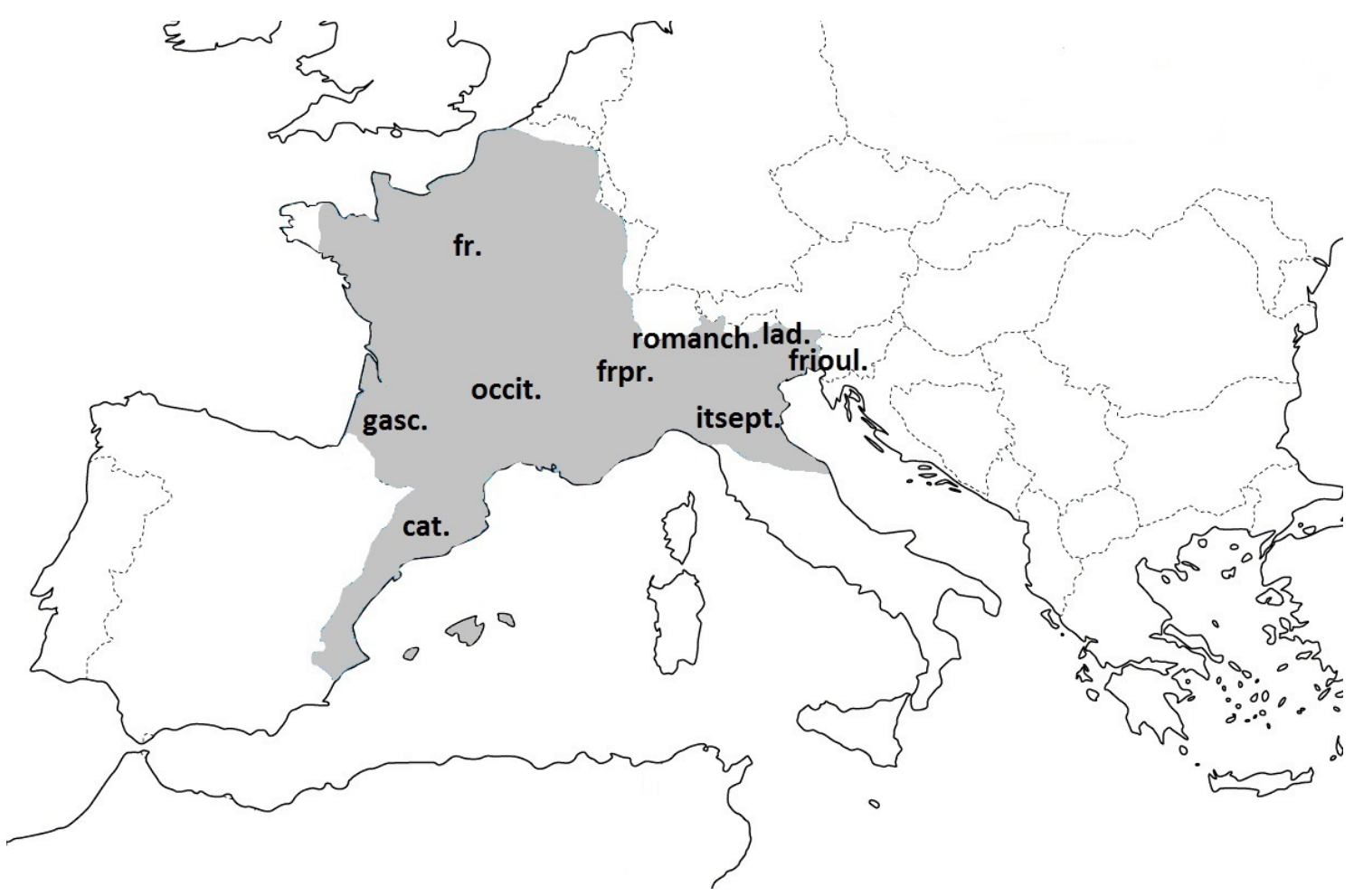

Carte 1 : fr. quérir et ses cognats

Postuler neuf créations idioromanes ${ }^{2}$ indépendantes est bien évidemment exclu : une telle coïncidence ne s'imagine tout simplement pas. Pour ce qui est de l'hypothèse d'une création idioromane (par exemple française) du Moyen Âge qui aurait été empruntée subséquemment par les autres langues romanes, elle nous paraît peu probable aussi, car plusieurs des idiomes concernés - notamment le frioulan, le ladin et le romanche - occupent des aires périphériques et isolées de la Romania : si quelques emprunts (entre le francoprovençal et l'italien septentrional ou entre l'occitan et le catalan, par exemple) ont pu intervenir, en imaginer huit paraîtrait vraiment excessif. L'hypothèse la plus en phase avec les données, marquées par une distribution aréale large, y compris dans des zones marginales et conservatrices, est dès lors celle d'un changement de classe flexionnelle intervenu à époque protoromane : sur la base de la comparaison entre les neuf cognats (italien septentrional cherire, frioulan cirî, ladin chirì etc.), on reconstruira leur ancêtre commun, protoroman */'kuعr-i-/ (infinitif : */kue'r-i-re/). Fr. quérir et ses congénères sont donc à considérer comme héréditaires.

Pour ce qui est de l'antériorité (absolue) des attestations de querre par rapport à celles de quérir, il semble s'agir d'une contingence documentaire : s'il est vrai, comme il ressort du tableau ci-dessous (dont les données sont issues de l'article */'kuعr-e-/ du DÉRom), que les idiomes qui connaissent les deux types attestent en général d'abord ‘querre $\urcorner$, ce qui frappe surtout, c’est la non correspondance des datations d'une langue à l'autre (ainsi l'occitan atteste par exemple déjà le type ‘quérir $\urcorner$ au même moment où 
querre apparaît seulement dans les textes français). La chronologie relative entre les témoignages écrits des deux types au sein d'une même langue, qui donne systématiquement - sauf cas indécidables (italien, frioulan) - l'avantage au type $\ulcorner$ querre $\urcorner$ (français, francoprovençal, occitan, gascon, catalan), semble donc plutôt indiquer que $\ulcorner$ querre $\urcorner$ représente le type non marqué par rapport à $\ulcorner q u e ́ r i r\urcorner$, le type qui aura eu le plus facilement accès à l'écrit. Ainsi, c'est une différence diastratique et/ou diaphasique qui pourrait opposer les deux types : au Moyen Âge (et peut-être déjà avant), le type $\ulcorner$ quérir $\urcorner$ aurait été relégué à un registre plus bas.

\begin{tabular}{|l|l|l|}
\hline Idiome & $\mathbf{1}^{\text {ère }}$ attestation du type querre & $\mathbf{1}^{\text {ère }}$ attestation du type quérir \\
\hline Italien & $c a 1230 / 1250$ & $1^{\text {ère }}$ moitié $13^{\mathrm{e}}$ siècle \\
\hline Frioulan & $c a 1416$ & $c a 1416$ \\
\hline Français & fin $11^{\mathrm{e}}$ siècle & 1327 \\
\hline Francoprovençal & $1220 / 1230$ & 1389 \\
\hline Occitan & $c a 1060$ & $1228 / 1229$ \\
\hline Gascon & 1125 & $15^{\mathrm{e}}$ siècle \\
\hline Catalan & fin $12^{\mathrm{e}}$ siècle & fin $13^{\mathrm{e}}$ siècle \\
\hline
\end{tabular}

Tableau 2 : comparaison des dates d'apparition des deux types dans les textes écrits

Pour l'ensemble de ces raisons, il n'y a donc pas de doute, du moins à notre avis, que le type quérir est héréditaire. De plus, le même changement de classe flexionnelle de la conjugaison en */'-e-/ à celle en */-'i-/ s'observe assez fréquemment en protoroman ( $c f$. par exemple Jatteau 2012/2013 in DÉRom s.v. */'фug-e-/ ou Grüner à paraître in DERom s.v. */'luk-e-/). Mais comment expliquer que des générations d'étymologistes romanistes et francisants soient passés à côté de cette analyse ? La réponse résidera sans aucun doute dans l'orientation trop excessive de l'ancienne méthode vers les données écrites : non seulement vers celles de l'ancien français, censées refléter sans distorsion aucune l'histoire du français (dans toute sa variation diasystémique) au Moyen Âge, mais aussi et surtout celles du latin. En effet, une des raisons, sans doute la raison centrale, de l'analyse unanime de fr. quérir comme une création idioromane résidera dans le fait que protoroman */'kuعr-i-/ ne connaît pas de corrélat en latin écrit de l'Antiquité. Du coup, seul quaerere était évoqué comme étymon (de querre), tandis que quérir était analysé comme une création française. Nous sommes donc en présence d'un cas typique où le latin écrit aura constitué une malédiction pour l'étymologie française et romane.

\section{Le latin écrit comme bénédiction}

Rappeler la bénédiction que le latin écrit de l'Antiquité représente pour l'étymologie romane relève du lieu commun le plus banal : des générations de romanistes (et de francisants) ont basé leurs pratiques sur ce postulat, en donnant comme éymons du lexique héréditaire des lexèmes latins (Bloch \& Wartburg $1968^{5}$ : an < annus, dent < dens, écrire < scrībere) ou, là où on ne dispose pas d'attestations écrites, des lexèmes que l'on pourrait attribuer à un «'fiddled with' classical Latin » (Buchi 2010b : 2 ; $c f$. Bloch \& Wartburg $1968^{5}$ : montagne $<[*]$ montānea ou rond $<*$ retundus).

Mais qu'en est-il du chercheur qui se place dans le cadre du paradigme de la grammaire comparée : puisque cette méthode amène à considérer comme illicite de placer «les mots du latin écrit de l'Antiquité » «à l'origine des mots héréditaires du français ou des autres langues (gallo)romanes » (cf. la citation de Chambon ci-dessus sous 1), condamne-t-elle à rejeter le latin écrit comme n'étant d'aucun secours pour l'étymologiste? Nous souhaitons affirmer avec vigueur qu'il n'en est rien : l'étymologiste romaniste peut en effet s'estimer heureux de pouvoir s'appuyer sur le témoignage du latin écrit, à 
condition de ne pas l'utiliser en lieu et place de la méthode comparative, mais de façon complémentaire. Utilisé de cette manière, le latin écrit nous semble en particulier pouvoir rendre deux services inestimables : d'une part, il peut aider à départager des hypothèses étymologiques entre lesquelles la grammaire comparée ne permet pas de trancher ( $c f$. ci-dessous 3.1), d'autre part, la comparaison entre un étymon reconstruit et son corrélat en latin écrit de l'Antiquité permet de mettre la main sur la variation diasystémique du latin global (cf. ci-dessous 3.2).

\subsection{Un exemple ponctuel : le genre de l'étymon de fr. gué}

L'étymologie de fr. gué pose un grave problème phonologique, dont les implications génétiques (influence germanique ou développement interne) sont de taille (cf. Möhren 2000 ; Alletsgruber 20112013 in DÉRom s.v. */'ßad-u/; Alletsgruber 2013). Dans le présent contexte, nous mettons toutefois de côté cette question pour nous intéresser plutôt aux propriétés morphosyntaxiques, et plus précisément au genre grammatical, de l'étymon de fr. gué et de ses cognats.

L'étymologie traditionnelle attribue soit le genre neutre à l'étymon de fr. gué, soit ne se prononce pas sur son genre :

- « aus fränkisch *wađzu a[lt]nord[isch] vađ`Furt', oder Kreuzung von lat[einisch] vadum [...] mit dem fränkischen Wort » (Gamillscheg $\left.1928^{1}-1969^{2}\right)$

- «Lat[in] vadum qui a pris en gallo-roman et en it[alien] le $w$ initial du mot apparenté *wađ du germ[anique]» (Bloch \& Wartburg $1932^{1}-1968^{5}$ );

- «francique * wad» (Dauzat, Dubois \& Mitterand 2007 [Dauzat 1938 ${ }^{1}$ : «lat[in] vadus, croisé avec le germ[anique] wad- $\gg])$

- «L[a]t[einisch] VADUM [...] im a[1t]n[ieder]fr[än]k[ischen] das neutrum » (von Wartburg 1964 in FEW 17, 440a, *WAĐ) ;

- «gallo-roman *wadu, croisement du lat[in] vadum et de son équivalent germ[anique] » (Picoche 1971 $\left.{ }^{1}-2002\right)$;

- «a[ncien] b[as] fr[anci]q[ue] *wađ» (TLF 1981);

- « francique * wad» (Robert historique $\left.1992^{1}-1998^{2}\right)$;

- « L[a]t[in] VĂDUM » (Möhren 1995 in DEAF G 1537)

- «franc[ique] *wad» (Baumgartner \& Ménard 1996).

On peut faire l'hypothèse que les dictionnaires qui n'attribuent pas de genre explicite à l'étymon le considèrent implicitement comme neutre, par mimétisme soit du latin écrit (en principe, un nom latin dont la forme citationnelle se termine par -um se dénonce comme neutre), soit, pour ceux qui posent un étymon germanique, des langues germaniques ( $c f$. le commentaire étymologique du FEW).

Pour ce qui est de l'étymologiste se situant dans le cadre de la méthode comparative, il ne pourra en aucun cas se satisfaire d'un raisonnement de ce type: pour lui, non seulement les propriétés phonologiques et sémantiques d'un étymon, mais aussi ses propriétés morphosyntaxiques, doivent être soigneusement reconstruites sur la base de la comparaison entre cognats romans. À cette raison théorique s'ajoute une raison pratique : comme on connait des cas où l'étymon d'une série de cognats romans présente un genre différent de celui de son corrélat en latin écrit (cf. par exemple fr. faim s.f. $<$ protorom. */'фamen/ s.n., face à lat. fames s.f., cf. Buchi/González Martín/Mertens/Schlienger 2012/2013 in DÉRom s.v. */'фamen/), la plus grande prudence est de mise : on considérera que par définition, le genre d'un étymon est inconnu au début du processus étymologique.

Il convient donc dans un premier temps d'établir la série de cognats autour de fr. gué. Sur la base des vingt idiomes obligatoires du DÉRom, elle comporte quatorze unités (cf. Alletsgruber 2011-2013 in DÉRom s.v. */'(ad-u/), l'istroroumain, le méglénoroumain, l'aroumain, l'istriote, le dalmate et le ladin n’étant pas représentés :

- sarde bádu s.m. ;

- dacoroumain vad s.n.; 


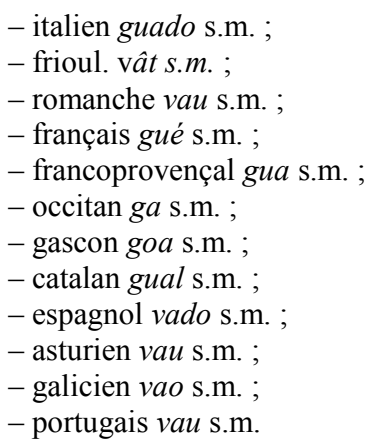

Treize cognats présentent le genre masculin, tandis que le cognat dacoroumain présente seul le genre neutre. Comment procéder pour reconstruire sur cette base le genre de leur ancêtre commun ? Il faut reconnaître que les manuels de grammaire comparée sont peu éloquents sur les opérations intellectuelles à mettre en œuvre lors de l'application de la méthode à la reconstruction des propriétés morphosyntaxiques des étymons. Anthony Fox, par exemple, considère qu'après la reconstruction phonologique, «the final step in the process of lexical reconstruction is the assignment of appropriate 'proto-meanings' to the items of the proto-lexicon" (Fox 1995: 112), éludant complètement toute mention de la reconstruction microsyntaxique. Mais il n'y a aucune raison de considérer que les critères classiques de la reconstruction comparative - majorité, directionnalité, traits partagés et économie ( $c f$. Campbell $\left.2004^{2}: 128-136\right)-$ ne s'appliquent pas ici.

Parmi ces quatre critères, celui concernant la majorité est le moins puissant : il ne doit être invoqué qu'en dernier recours, notamment en raison de l'articulation interne que présentent souvent les familles linguistiques : " majority rule may not work if some of the languages are more closely related to one another » (Campbell 2004² : 131). Dans le cas qui nous occupe, les quatorze cognats en présence n'ont pas tous le même statut au sein de l'arbre phylogénétique roman: comme on admet en général (Klinkenberg 1999² : 135 ; Vallejo 2012 : 454 ; cf. aussi Hall 1950), les différents idiomes romans se rattachent génétiquement à deux principales branches: d'une part au protosarde, d'autre part au protoroman continental. Du coup, la question de la majorité se pose dans des termes moins évidents que ce que l'on pourrait penser (treize masculins face à un seul neutre) : en tenant compte de la relative unité primitive de la branche continentale, on peut admettre un rapport de force de 1 à 1 entre le masculin (protosarde) et le neutre ou le masculin (protoroman continental).

Le critère assurément le plus puissant dans notre contexte est celui de la directionnalité, puisqu'il est avéré que lors du passage du latin aux langues romanes, le neutre a presqu'entièrement disparu, pour se maintenir seulement dans la branche roumaine ( $c f$. par exemple Lausberg $1976^{2}: 2: 22$ ). Ayant un statut clairement récessif, le genre neutre se trouve donc être privilégié lors de la reconstruction. C'est pour cette raison, par exemple, que le DÉRom reconstruit un étymon neutre pour fr. ail (Reinhardt 2010-2012 in DÉRom s.v. */'ali-u/), foin (Reinhardt 2008-2012 in DÉRom s.v. */'фen-u/ */'фعn-u/) ou encore mô̂t (Delorme 2011/2012 in DÉRom s.v. */'must-u/).

Mais le genre neutre est productif en dacoroumain (Graur 1928 : 251-252, 256-257, 260), de sorte que le caractère récessif du neutre roman ne doit pas être prétexte à la reconstruction systématique d'un neutre dès que l'on est en présence d'un cognat dacoroumain neutre. Afin de se prémunir contre le danger d'un neutre non héréditaire, de création idioromane, il convient dès lors d'exiger qu'au moins deux cognats romans relèvent du genre neutre pour attribuer le genre neutre à leur ancêtre commun. À côté du dacoroumain, de tels cognats neutres (ou, selon la terminologie, alternants) peuvent notamment être relevés dans les variétés sud-danubiennes du roumain (istroroumain, méglénoroumain, aroumain) et en italien (type braccio s.m.sg. 'bras'/braccia s.f.pl. 'ensemble des deux bras' ; $c$. aussi Loporcaro \& Paciaroni 2011 pour des traces d'un ancien neutre dans certains dialectes italiens).

Or, lors de l'étymologisation de fr. gué et de ses congénères, l'équipe du DÉRom n'a pas réussi à trouver un second cognat neutre à côté de dacoroum. vad. Ce critère n'est donc pas non plus déterminant ici. 
Le troisième critère, celui des traits partagés, ne permet pas non plus de départager les deux hypothèses, puisque le trait partagé entre 'substantif masculin' et 'substantif neutre' est 'substantif', particularité de l'étymon qui ne pose nullement problème.

Reste le dernier critère, qui porte sur l'économie des changements à invoquer. Ce critère semble a priori favoriser l'hypothèse d'un masculin : alors qu'un étymon neutre impose de postuler deux changements indépendants (neutre $>$ masculin en sarde et neutre $>$ masculin dans la branche italo-occidentale, sousbranche du protoroman continental), un étymon masculin ne présuppose qu'un seul changement (masculin $>$ neutre en roumain). Toutefois, ce raisonnement se dénonce comme erroné une fois que l'on fait intervenir dans l'équation le fait que la perte du neutre est systématique dans la totalité des idiomes romans hormis le roumain ( $c f$. ci-dessus).

Force est donc de constater que nous nous trouvons ici en face d'un cas d'aporie au sein de l'étymologie reconstructrice : la méthode de la grammaire comparée ne permet pas d'assigner avec sûreté un genre particulier à l'étymon de fr. gué. Il est vrai que l'on peut exclure un féminin, et que le critère de la directionnalité semble concéder un léger avantage au neutre (même si ce point prête probablement à discussion). Pour cette raison, l'étymon */'Bad-u/ du DÉRom porte la catégorie grammaticale 's.[n. ou m.]' (= substantif neutre ou masculin).

Le lecteur aura été sensible aux potentialités qui s'ouvrent au latin écrit dans de tels cas: quand la méthode de la grammaire comparée s'avère incapable d'aller au bout du processus reconstructif, la comparaison du résultat, incomplet, de la reconstruction avec les données du latin écrit de l'Antiquité sera des plus intéressantes. En l'occurrence, Julia Alletsgruber, l'auteure de l'article du DÉRom, s'exprime ainsi dans le commentaire :

\begin{abstract}
Le corrélat du latin écrit de I [= dacoroum. vad s.n.], uadum, -i s.n. "gué », est connu durant toute l'Antiquité (dp. Plaute [* ca $254-\dagger 184]$, OLD), celui de II. [= fr. gué s.m. etc.], uadus, $-i$ s.m. «id. », est attesté sporadiquement entre Varron (* $116-$ $\dagger 27$ ) et Fronton (* $c a 100-\dagger c a 170$, tous les deux OLD). La reconstruction d'un neutre originel, que la seule méthode comparative ne permet pas d'établir de façon définitive, trouve donc dans le corrélat de la langue écrite un appui solide.
\end{abstract}

À noter que la formulation de la déromienne est prudente : elle ne laisse pas penser que les données latines permettraient de départager à elles seules deux hypothèses entre lesquelles la grammaire comparée hésiterait ${ }^{3}$. En réalité, aucune des deux méthodes n'est capable, à elle seule, d'assigner de façon définitive un genre à l'étymon de fr. gué: si la reconstruction comparative en est incapable pour les raisons évoquées ci-dessus, la philologie latine n'en a pas non plus les moyens, car il peut exister des différences entre les propriétés morphosyntaxiques d'un lexème du latin oral et celles du même lexème en latin écrit ( $c f$. ci-dessus le cas de l'étymon de fr. faim). En revanche, le romaniste s'estimera heureux de disposer des témoignages du latin écrit, car la mise en commun des résultats des deux approches, tous les deux incomplets, permet de toucher du doigt la réalité d'une protolangue d'une manière non égalée dans d'autres domaines linguistiques.

\title{
3.2 Et plus généralement : accès à la variation diasystémique du latin
}

Le cas de fr. gué nous a permis d'apprécier la contribution ponctuelle que le latin écrit de l'Antiquité peut fournir au traitement d'un problème de morphologie flexionnelle qui frappe un étymon protoroman. Dans ce qui suit, nous voudrions plaider pour une conception de l'étymologie romane (et donc française) au sein de laquelle les données du latin écrit revêtent une importance non pas ponctuelle, mais structurelle, c'est-à-dire (potentiellement) systématique.

En effet, l'étymologie romane se distingue de toutes les autres branches de la science étymologique par le fait qu'elle peut s'appuyer sur les résultats de recherche de deux méthodes complémentaires. Comme l'une ne pourra toujours reconstruire, par définition, que des variétés orales, et que l'autre, par définition, ne pourra donner à voir que des variétés écrites, le premier constat qui s'impose, c'est que la confrontation des résultats des deux méthodes permet de révéler immédiatement des éléments de variation 
diamésique du latin global. Après avoir reconstruit, comme ancêtre commun d'italien montagna, français montagne, occitan montanha etc., protoroman */mon't-ani-a/ s.f. 'territoire caractérisé par d'importantes élévations du terrain ; importante élévation de terrain', dérivé en */-'ani-/ (suffixe à valeur collective) de */'mont-e/ s.m. « importante élévation de terrain », Victor Celac termine ainsi le commentaire de son article par l'observation suivante : «le latin écrit de l'Antiquité ne connaît pas de corrélat de protorom. */mon't-ani-a/. Du point de vue diasystématique ("latin global"), */mon't-ani-a/ est donc à considérer comme un particularisme (oralisme) de la variété B (basse)» (Celac 2012/2013 in DÉRom s.v. */mon't-ani-a/).

Mais l'intérêt des données du latin écrit ne s'arrête pas là. Comme la méthode comparative donne plutôt (mais pas tout à fait exclusivement) accès à des variétés d'immédiat communicatif et que la philologie latine donne plutôt (mais pas tout à fait exclusivement) à voir des variétés de distance communicative ${ }^{4}$, la comparaison des résultats des deux peut dépasser le seul axe diamésique pour porter sur la variation diaphasique, voire diastratique. Pour en revenir à quérir, l'article du DÉRom qui en traite est structuré comme suit :

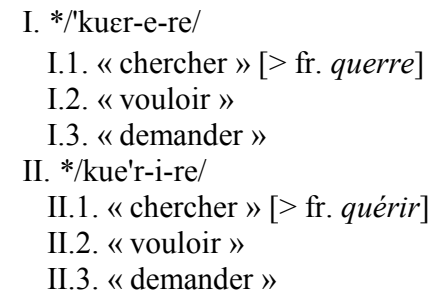

Le commentaire de cet article introduit les données du latin écrit de la manière suivante :

Le corrélat de I.1., lat. quaerere v.tr. «chercher» (dp. Plaute [* ca $254-\dagger 184]$, OLD), et celui de I.3., lat. quaerere v.tr. « demander » (dp. Varron [* $116-\uparrow 27]$, OLD), sont connus durant toute l'Antiquité, tandis que le latin écrit de l'Antiquité ne connaît pas de corrélat relevant de la flexion en */-'i-/ ni présentant le sens « vouloir $»^{15}$ (types I.2. et II.).

$\mathrm{Du}$ point de vue diasystématique ("latin global"), la flexion en */-'i-/ et le sens «vouloir » sont à considérer comme des particularismes (oralismes) de la variété $\mathrm{B}$ qui n'ont eu aucun accès à la variété $\mathrm{H}$ : la diversité de la première s'oppose à l'unité de la seconde.

15. On relève dans les textes latins, surtout à partir d'auteurs chrétiens comme Tertullien, des emplois de quaerere en fonction modale qui préfigurent l'apparition du sens « vouloir » (cf. Maggiore,LVLT 10, 6).

On entrevoit là l'immense bénéfice que l'étymologiste peut tirer de la consultation et de l'utilisation raisonnée des données du latin écrit de l'Antiquité : en posant, comme étymon de français quérir, «protoroman régional */'kuer-i-/ v.tr. 's'efforcer de trouver' », le chercheur s'acquitte, certes, de sa mission immédiate. Toutefois, pour autant que l'on accepte l'idée que les étymologies particulières nourrissent le projet plus global de la reconstruction des structures lexicales de la protolangue, ainsi que celle, peut-être plus ambitieuse encore, que les études romanes ont leur pierre à apporter à la connaissance du diasystème latin ${ }^{5}$, on ne peut pas s'arrêter là : il convient aussi, dans un premier temps, de se poser la question du corrélat dans la langue écrite de l'étymon reconstruit ( $c f$. le premier paragraphe de la citation ci-dessus), puis de comparer les particularités - en l'occurrence, flexionnelles et sémantiques - des deux unités ainsi posées dans le but d'accéder à la variation interne du diasystème latin ( $c f$. le second paragraphe de la citation ci-dessus).

\section{Conclusion}

Quel sera donc, à l'issue de cette brève réflexion, le statut que nous assignerions, en étymologie héréditaire romane (et donc française), au latin écrit de l'Antiquité ? En réalité, notre expérience de 
praticiens nous incite à nous méfier de deux écueils à éviter : d'une part, le romaniste ne doit pas se laisser aveugler par l'abondance des données du latin écrit au point de se fermer à la reconstruction comparative comme méthode de connaissance de l'ancêtre commun des idiomes romans ; d'autre part, il doit à tout prix éviter de tomber dans le travers inverse, qui consisterait à ne pas considérer du tout ces données extrêmement précieuses. À l'issue du processus de reconstruction comparative, une confrontation de l'étymon ainsi établi avec la manne que constituent les attestations latines s'avère en effet des plus instructives, non seulement dans les cas où la reconstruction n'aboutit pas à un résultat univoque (exemple de gué), mais de façon potentiellement systématique, dans le but de toucher du doigt la variation interne du latin global (exemples de montagne et de quérir). C'est dans ce sens qu' " it is a special privilege of Romance philologists that they are not compelled to rely entirely upon reconstruction » (Elcock $1975: 33$ ) : non pas, comme cet auteur semble le penser (la citation forme la première phrase du paragraphe «Direct evidence for Vulgar Latin »), parce que les données du latin écrit donneraient accès à elles seules à la connaissance de traits de l'ancêtre commun des parlers romans, mais parce que, conjuguées aux résultats de la reconstruction comparative, elles permettent de donner à voir des pans entiers de la variation diasystémique interne d'une langue dont leur ancêtre commun représente une partie constitutive.

Arrivés à ce stade de la démonstration, d'aucuns seront peut-être tentés de militer en faveur d'une utilisation conjointe des deux principales méthodes de connaissance du latin global, la reconstruction comparative et la philologie latine, pour l'établissement des étymons (voire en faveur d'une utilisation conjointe de toutes les méthodes disponibles, donc y compris, par exemple, du témoignage indirect que fournissent les emprunts au latin dans les langues non romanes). Dans cette hypothèse, l'étymon de fr. gué, par exemple, ne serait pas étiqueté «s.[n. ou m.]», mais «s.n.», sur la foi des abondantes attestations écrites témoignant de son genre neutre. Si, dans le cadre du DÉRom, nous renonçons à adopter une telle présentation lexicographique, c'est qu'elle nous semble incompatible avec l'analyse linguistique qui sous-tend notre approche. En effet, dans notre conception, ce n'est pas le latin global dans son ensemble qui représente l'ancêtre commun des parlers romans, mais seulement ses variétés orales (Buchi 2013b) : la quantité vocalique, par exemple, est étrangère au protoroman, et le futur synthétique ne représente pas un tiroir de son système verbal. Dans la phrase «Latin, the (near-)ancestor of the Romance languages » (Hock 1991: 611), où Latin renvoie au latin écrit, le modulateur near- est important (et ne devrait d'ailleurs pas être placé entre parenthèses). Dès lors, un attachement à une démarche épistémologiquement irréprochable nous conduit à n'attribuer aux étymons, qui sont censés représenter les ancêtres communs d'une série de cognats romans, que les traits que nous pensons être en mesure de leur attribuer sur la base de la seule reconstruction comparative - y compris dans les cas, comme dans celui de */'ßad-u/, où le doute (en l'occurrence, celui qui frappe le genre du lexème dans les variétés orales du latin) est réduit à la portion congrue. Nous espérons ainsi échapper à une certaine circularité qui caractérise la méthode traditionnelle que Joseph Herman avait déjà eu l'occasion d'épingler :

[...] les procédés heuristiques propres à la méthode comparative se présentent presque toujours intégrés à une démarche historique qui suit la marche du temps, et qui se fonde sur une analyse linguistique des documents disponibles. C'est sans doute un peu regrettable: le non romaniste distingue difficilement ce que nous savons grâce aux particularités du latin tardif et ce que nous postulons sur la base des méthodes comparatives [...]. (Herman $2001: 716)$.

\section{Références bibliographiques}

Alletsgruber, J. (2013). À la recherche d'une étymologie panromane : lexique héréditaire roman et influence du superstrat germanique dans le DÉRom (Dictionnaire Étymologique Roman) : le cas de */'ßad-u/ */'uad-u/ "gué". In : Chabrolle-Cerretini, A.-M. (éd.), Romania : réalité(s) et concepts. Actes du colloque international des 6 et 7 octobre 2011, Université Nancy 2. Limoges : Lambert et Lucas, 123-131.

Baumgartner, E. \& Ménard, Ph. (1996). Dictionnaire étymologique et historique de la langue française. Paris: Librairie Générale Française. 
Benarroch, M. (2013). Latin oral et latin écrit en étymologie romane : l'exemple du DÉRom (Dictionnaire Étymologique Roman). In : Carreira, M. H. Araújo (éd.), Les Rapports entre l'oral et l'écrit dans les langues romanes. Saint-Denis : Université Paris 8, 127-158.

Bloch, O. \& Wartburg, W. von $\left(1968^{5}\left[1932^{1}\right]\right)$. Dictionnaire étymologique de la langue française. Paris : Presses Universitaires de France.

Bourciez, É. \& Bourciez, J. (1967). Phonétique française. Étude historique. Paris : Klincksieck.

Buchi, É. (2010a). Pourquoi la linguistique romane n'est pas soluble en linguistiques idioromanes. Le témoignage du Dictionnaire Étymologique Roman (DÉRom). In : Alén Garabato, C. et al. (éd.), Quelle linguistique romane au $X X I^{e}$ siècle? Paris : L'Harmattan, 43-60.

Buchi, É. (2010b). Where Caesar's Latin does not belong: a comparative grammar based approach to Romance etymology. In : Brewer, Ch. (éd.), Selected Proceedings of the Fifth International Conference on Historical Lexicography and Lexicology held at St Anne's College, Oxford, 16-18 June 2010, Oxford, Oxford University Research Archive (http://ora.ox.ac.uk/objects/uuid\%3A237856e6-a327-448b-898c-cb1860766e59).

Buchi, É. (2012). Des bienfaits de l'application de la méthode comparative à la matière romane : l'exemple de la reconstruction sémantique. In : Vykypěl, B. \& Boček, V. (éd.), Methods of Etymological Practice. Prague : Nakladatelství Lidové noviny, 105-117.

Buchi, É. (2013a). Cent ans après Meyer-Lübke: le Dictionnaire Étymologique Roman (DÉRom) en tant que tentative d'arrimage de l'étymologie romane à la linguistique générale. In : Casanova Herrero, E. \& Calvo Rigual, C. (éd.), Actas del XXVI Congreso Internacional de Lingüística y de Filología Románicas (Valencia 2010). Berlin/New York : De Gruyter, 1, 141-147.

Buchi, É. (2013b). Qu'est-ce que le protoroman? La contribution du DÉRom (Dictionnaire Étymologique Roman). Communication inédite présentée lors du XXVIII ${ }^{\mathrm{e}}$ Romanistisches Kolloquium (Rauischholzhausen, 30 mai- ${ }^{\text {er }}$ juin 2013).

Buchi, É. (2013c). Les langues romanes sont-elles des langues comme les autres? Ce qu'en pense le DÉRom. Communication inédite présentée au XXVII ${ }^{\mathrm{e}}$ Congrès international de linguistique et de philologie romanes (Nancy, 15-20 juillet 2013), section 1 : Linguistique générale/linguistique romane.

Buchi, É. (à paraître). Etymological dictionaries. In : Durkin, Ph. (éd.), The Oxford Handbook of Lexicography. Oxford : Oxford University Press.

Buchi, É., Chauveau, J.-P., Gouvert, X. \& Greub, Y. (2010). Quand la linguistique française ne saurait que se faire romane : du neuf dans le traitement étymologique du lexique héréditaire. In : Neveu, F. et al. (éd.), Congrès Mondial de Linguistique Française - CMLF 2010. Paris, Institut de Linguistique Française (http://dx.doi.org/10.1051/cmlf/2010025), 111-123.

Buchi, É., González Martín, C., Mertens, B. \& Schlienger, C. (à paraître). L’étymologie de FAIM et de FaminE revue dans le cadre du DÉRom. Le français moderne.

Buchi, É. \& Schweickard, W. (2009). Romanistique et étymologie du fonds lexical héréditaire : du REW au DÉRom (Dictionnaire Étymologique Roman). In: Alén Garabato, C. et al. (éd.), La Romanistique dans tous ses états. Paris : L'Harmattan, 97-110.

Buchi, É. \& Schweickard, W. (2011a). Sept malentendus dans la perception du DÉRom par Alberto Vàrvaro. Revue de linguistique romane, $75,305-312$.

Buchi, É. \& Schweickard, W. (2011b). Ce qui oppose vraiment deux conceptions de l'étymologie romane. Réponse à Alberto Vàrvaro et contribution à un débat méthodologique en cours. Revue de linguistique romane, 75, 628-635.

Buchi, É. \& Schweickard, W. (2013). Per un'etimologia romanza saldamente ancorata alla linguistica variazionale : riflessioni fondate sull'esperienza del DÉRom (Dictionnaire Étymologique Roman). In: Boutier, M.-G., Hadermann, P. \& Van Acker, M. (éd.), La variation et le changement en langue (langues romanes). Helsinki : Société Néophilologique, 47-60.

Campbell, L. (2004² [1998 $\left.\left.{ }^{1}\right]\right)$. Historical Linguistics. An Introduction. Cambridge : MIT Press.

Chambon, J.-P. (2007). Remarques sur la grammaire comparée-reconstruction en linguistique romane (situation, perspectives). Mémoires de la Société de linguistique de Paris, 15, 57-72. 
Chambon, J.-P. (2010). Pratique étymologique en domaine (gallo)roman et grammaire comparée-reconstruction. À propos du traitement des mots héréditaires dans le $T L F$ et le $F E W$. In : Choi-Jonin, I., Duval, M. \& Soutet, O. (éd.), Typologie et comparatisme. Hommages offerts à Alain Lemaréchal. Louvain : Peeters, 61-75.

Chambon, J.-P. (à paraître). Réflexions sur la reconstruction comparative en étymologie romane : entre Meillet et Herman. In : Gleßgen, M.-D. \& Schweickard, W. (éd.), Étymologie romane. Objets, méthodes et perspectives. Strasbourg : Société de linguistique romane/ELiPhi.

Dauzat, A., Dubois, J. \& Mitterand, H. (2007 $\left.\left[1938^{1}\right]\right)$. Dictionnaire étymologique et historique du français. Paris : Larousse.

DEAF = Baldinger, K. et al. (1974-). Dictionnaire Étymologique de l'Ancien Français. Québec/Tübingen/Paris : Presses de l’Université Laval/Niemeyer/Klincksieck.

DÉRom = Buchi, É. \& Schweickard, W. (dir.) (2008-). Dictionnaire Étymologique Roman (DÉRom). Nancy: ATILF (http://www.atilf.fr/DERom)

Dworkin, S. N. (à paraître). Etymology. In : International Encyclopedia of the Social \& Behavioral Sciences. Amsterdam : Elsevier.

Elcock, W. D. (1975 [1960]). The Romance Languages. Londres : Faber \& Faber.

FEW = Wartburg, W. von et al. (1922-2002). Französisches Etymologisches Wörterbuch. Eine darstellung des galloromanischen sprachschatzes (25 vol.). Bonn/Heidelberg/Leipzig-Berlin/Bâle : Klopp/Winter/Teubner/Zbinden.

Fox, A. (1995). Linguistic Reconstruction. An Introduction to Theory and Method. Oxford : Oxford University Press.

Gamillscheg, E. (1969² [1928 $\left.\left.{ }^{1}\right]\right)$. Etymologisches Wörterbuch der französischen Sprache. Heidelberg : Winter.

Gdf = Godefroy, F. (1881-1895). Dictionnaire de l'ancienne langue française et de tous ses dialectes du $I X^{e}$ au $X V^{e}$ siècle (8 vol.). Paris : Vieweg.

Graur, A. (1928). Les substantifs neutres en roumain. Romania, 54, 249-260.

Hall, R. A. Jr. (1950). The reconstruction of Proto-Romance. Language, 26, 6-27.

Herman, J. (2001). Linguistique comparée. In: Holtus, G., Metzeltin, M. \& Schmitt, C. (éd.), Lexikon der Romanistischen Linguistik (LRL). Tübingen : Niemeyer, I/2, 704-718.

Hock, H. H. (1991² [1986¹]). Principles of Historical Linguistics. Berlin : Mouton De Gruyter.

Klein, H.-W. (1961). Contribution à la différentiation sémantique de la Romania (I). Orbis, 10, 144-156.

Klinkenberg, J.-M. (1999² [1994 $])$. Des langues romanes. Introduction aux études de linguistique romane. Paris/Bruxelles : Duculot.

Koch, P. \& Oesterreicher, W. (2008). Comparaison historique de l'architecture des langues romanes. In : Ernst, G. et al. (éd.) : Histoire linguistique de la Romania. Manuel international d'histoire linguistique de la Romania. Berlin/New York : De Gruyter, 3, 2575-2610.

Kramer, J. (2011). Latein, Proto-Romanisch und das DÉRom. Romanistik in Geschichte und Gegenwart, 17, 195206.

Lausberg, H. (1976 $\left.{ }^{2}\left[1971^{1}\right]\right)$. Linguistica romanza (2 vol.). Milan : Feltrinelli.

Loporcaro, M. \& Paciaroni, T. (2011). Four-gender systems in Indo-European. Folia Linguistica, 45, 389-433.

Maggiore, M. (à paraître). Note di etimologia romanza a margine dell'articolo */'kuєr-e-/ (quaerĕre) del Dictionnaire Etymologique Roman. In : Molinelli, P. et al. (éd.), Actes du colloque international "Latin vulgaire - Latin tardif $X^{\prime \prime}$, Bergame, 4-9 septembre 2012. Bergame : Sestante Edizioni.

Meyer-Lübke, W. (1890-1906). Grammaire des langues romanes (4 vol.). Paris : Welter.

Möhren, F. (2000). « Guai victis ! » Le problème du gu initial roman. Medioevo romanzo, 24, 5-81.

Möhren, F. (2012). Édition, lexicologie et l'esprit scientifique. In : Trotter, D. (éd.), Present and future research in Anglo-Norman. Proceedings of the Aberystwyth Colloquium, 21-22 July 2011. Aberystwyth: The Anglo-Norman Online Hub, 1-13. 
Picoche, J. (2002 [1971 $\left.\left.{ }^{1}\right]\right)$. Dictionnaire étymologique du français. Paris : Le Robert.

$\mathrm{REW}_{3}=$ Meyer-Lübke, W. (1930-1935 $\left.{ }^{3}\left[1911-1920^{1}\right]\right)$. Romanisches Etymologisches Wörterbuch. Heidelberg: Winter.

Robert historique = Rey, A. (dir.) $\left(1998^{2}\left[1992^{1}\right]\right)$. Dictionnaire historique de la langue française. Paris : Le Robert.

TLF = Imbs, P. \& Quemada, B. (1971-1994). Trésor de la langue française. Dictionnaire de la langue du XIX et du XX siècle (1789-1960) (16 vol.). Paris : Éditions du CNRS/Gallimard.

Vallejo, J. M. (2012). Del proto-indoeuropeo al proto-romance. Romance Philology, 66, 449-467.

Vàrvaro, A. (2009). Tra latino e lingue romanze. Gli studi di J.N. Adams sul latino e la linguistica romanza. Revue de linguistique romane, 73, 601-622.

Vàrvaro, A. (2011a). Il DÉRom : un nuovo REW? Revue de linguistique romane, 75, 297-304.

Vàrvaro, A. (2011b). La 'rupture épistémologique' del DÉRom. Ancora sul metodo dell'etimologia romanza. Revue de linguistique romane, $75,623-627$.

\footnotetext{
${ }^{1}$ Nos remerciements les plus chaleureux s'adressent à Pascale Baudinot (Nancy), Jérémie Delorme (Le GrandBornand), Steven N. Dworkin (Ann Arbor), Yan Greub (Nancy), Laure Grüner (Neuchâtel), Ulrike Heidemeier (Nancy) et Mihaela-Mariana Morcov (Bucarest), ainsi qu'à deux relecteurs anonymes, pour leurs réactions stimulantes à une première version de ce texte.

${ }^{2}$ Pour ce terme technique, dont le sens est 'relatif à une langue romane particulière', $c f$. Buchi \& Schweickard (2009 : 101).

${ }^{3}$ En cela, Julia Alletsgruber rejoint l'attitude de Jean-Pierre Chambon: «la confrontation avec les données philologiques n'est à envisager qu'en dernier lieu. Dans l'optique ici retenue, cette opération ne possède du reste qu'une portée assez réduite. L'examen de la documentation écrite ne peut jamais que confirmer certains des résultats de la reconstruction (généralement les plus sûrs). Il n'est pas en mesure de les démentir; il n'est pas en droit de les modifier. Encore moins peut-il émettre [...] la prétention de tester et de valider la méthode comparative en s'instituant en juge, placé au-dessus de celle-ci » (Chambon 2007 : 69).

${ }^{4}$ Pour les notions d'immédiat communicatif et de distance communicative, $c f$. Koch \& Oesterreicher (2008).

${ }^{5}$ Selon la formule d'Alberto Vàrvaro, le diaystème latin est « una realtà unica osservata da due punti di vista diversi (in retrospettiva ed in prospettiva)» (Vàrvaro $2009: 602$ ).
} 